\title{
Loss to Follow-Up among a Group of Patients with HIV and Severe Mental Illness in South Africa
}

\author{
Todd Wagner ${ }^{1}$, Jennifer Furin ${ }^{2 *}$, Barbara Gripshover ${ }^{3}$, Yasmien Jeenah ${ }^{4}$, Greg Jonsson ${ }^{4}$ \\ ${ }^{1}$ Case Western Reserve University School of Medicine, Department of Family Medicine \& Community \\ Health, Cleveland, USA \\ ${ }^{2}$ Case Western Reserve University School of Medicine, TB Research Unit, Cleveland, USA \\ ${ }^{3}$ Case Western Reserve University School of Medicine, Division of Infectious Diseases, Cleveland, USA \\ ${ }^{4}$ Department of Psychiatry, University of the Witwatersrand, Johannesburg, South Africa \\ Email: ${ }^{\text {jjj38@case.edu }}$
}

Received 18 November 2013; revised 18 December 2013; accepted 25 December 2013

Copyright (C) 2014 by authors and Scientific Research Publishing Inc.

This work is licensed under the Creative Commons Attribution International License (CC BY).

http://creativecommons.org/licenses/by/4.0/

(c) (i) Open Access

\begin{abstract}
Background: Persons with HIV and severe mental illness face numerous barriers in antiretroviral treatment adherence. More information is needed on reasons for loss of follow-up in this population. Methods: A retrospective analysis was conducted on adult HIV patients with a history of mental illness enrolled at an urban HIV clinic in Johannesburg, South Africa who discontinued care. Results: $\mathbf{2 4 . 8 \%}$ of adult patients in the clinic discontinued follow-up during the study period. Of those discontinuing follow-up, 48 were successfully traced by home visits. Among this group, 21 $(43.8 \%)$ were not engaged in care, $12(25.0 \%)$ had transferred care, $9(18.8 \%)$ were deceased, 3 $(6.2 \%)$ had relocated, and $3(6.2 \%)$ were missing. Transportation costs and distance, conflicts with work/school schedule, and confusion regarding when to return were the most frequently cited reasons for discontinuing follow-up. Conclusions: Although almost $25 \%$ of patients were lost to follow up, overall rates of retention in care for these patients were similar to those seen in other HIV-infected populations and higher than those seen among patients with psychiatric disease. Tracing patients through home visits proved to be an effective means to confirm the magnitude of patients lost to follow up, ascertain their outcomes, and elucidate the reasons for discontinuing care.
\end{abstract}

Keywords

HIV; Mental Illness; Loss of Follow-Up; South Africa

\footnotetext{
${ }^{*}$ Corresponding author.
} 


\section{Introduction}

The introduction of highly-active antiretroviral therapy (HAART) in the mid-1990s dramatically altered the course of AIDS pandemic around the world [1], allowing persons with HIV to live relatively disease-free lives as long as they are able to adhere to their antiretroviral regimens [2]. Although introduction of these medications into high-burden, resource-poor settings was slow to commence and has been inadequate to meet global needs [3] [4], significant portions of populations around the world now have access to antiretroviral therapy (ART) [5]. Key to achieving good outcomes from ART is the ability to remain in medical care on a chronic, long-term basis [6] [7].

The country of South Africa has a high HIV seroprevalence with an estimated 18\% of the general population infected with HIV [8] [9]. One group which has been disproportionately affected is individuals with mental illness [10]. An estimated 26.5\% of individuals with mental illness in South Africa have HIV [11]. Mental illness has been shown to increase the risk of acquiring HIV, and some studies have shown that persons with mental illness may have worse treatment outcomes [12]-[14]. Multiple factors associated with these poor outcomes have been described, including stigma [15], increased barriers in accessing care, and poor insight and judgment associated with active mental illness [16]. Many of these issues can be alleviated through integrated care programs [17] [18] which have been highly successful in treating persons with HIV and severe mental illness [19] [20]. Even in the best programs, however, a substantial proportion of patients with HIV and mental illness are at risk for becoming "lost to follow up" [21].

Once patients are lost to medical care, it is likely that they are no longer on ART [22]. This not only leads to poor clinical outcomes among such individuals but can also lead to the development of antiviral drug resistance and contribute to the ongoing spread of HIV in the community [23] [24]. Furthermore, since the initial ART regimen offers the best chance of viral suppression, immunologic recovery, and clinical stability, it is imperative that individuals be quickly integrated into a medical system that meets their needs [25]. This paper will describe the rate of and reasons for loss of follow-up among a population of patients with HIV and severe mental illness receiving care through an integrated program for persons with a dual diagnosis

\section{Setting}

This study took place at the Luthando Neuropsychiatric HIV Clinic in Soweto, Johannesburg, South Africa. The clinic was founded in 2008 as part of the Chris Hani Baragwanath Academic Hospital and serves the population of Soweto, a community in the southwestern part of Johannesburg with an estimated population of 3 million. The goal of the clinic is to provide a "one stop shop" for persons living with HIV and severe mental illness. Here, they receive integrated medical care for their HIV, opportunistic infections (including TB), and psychiatric disease. The clinic also offers group therapy, income generation groups, occupational therapy, and creative writing [26]. It currently serves a population of more than 1000 individuals and is staffed by 2 full-time and 2 part-time psychiatrists, 1 professional nurse, 1 enrolled nurse, and 2 administrative staff members. All patient records are maintained electronically, and include a large database capturing clinically relevant materials, such as loss of follow-up.

\section{Methods}

The study had two main components: 1) a retrospective data analysis of individuals identified as lost to follow up, and 2) tracing home visits. A retrospective analysis was conducted on patients entered into the database from July 2, 2010 through September 15, 2011. Patients discontinuing follow-up, defined as $\geq 6$ months since last clinic visit, were identified and studied further. Baseline demographic and clinical characteristics were reviewed from available medical records.

The second part of the study consisted of interviews with patients who were identified as being lost to follow up in the database. Individuals were traced via a home visit using contact information listed in the clinic registry or patient's medical record. Upon contact, an interview was conducted to determine the health status of the patient and reasons for loss of follow-up. During the study period 561 patients were entered into the database, of which 139 were identified as lost to follow up. Of these individuals, 48 were successfully traced and 36 agreed to be interviewed for the study (Figure 1). Interviews were conducted in the study participant's native language using an open-ended interview guide. 


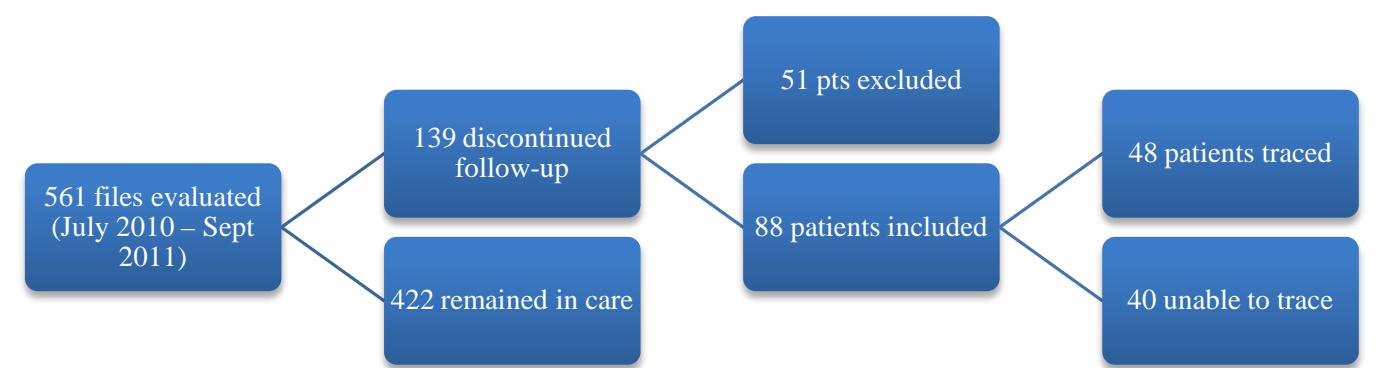

Figure 1. Flow of participants.

Ethical approval for this study was provided by the Human Research Ethics Committee at University of Witswatersrand (Johannesburg, South Africa) and the Institutional Review Board at Case Western Reserve University (Cleveland, OH, USA).

\section{Results}

Of the 561 patients whose records were reviewed, 139 (24.8\%) were identified as lost to follow up. Of those patients, 51 were excluded due to incomplete or unavailable medical records. From the remaining 88 patients, 48 home visits were successfully conducted. Among those individuals, 36 were traced alive, 9 deceased, and 3 missing. Of the 48 patients identified as lost to follow up and traced through home visits, $62.5 \%$ were female, mean age was 33 years, and median baseline CD4 count was 143 cells/ $\mu \mathrm{L}$. This was consistant with the loss of follow-up group as a whole, of which $64.8 \%$ were female with a mean age of 37.2 years. Additional demographic and clinical characteristics are described in Table 1.

Regarding the outcome evaluated at the home visits, 21 (43.8\%) were not currently engaged in care; 12 (25.0\%) had transferred care to another clinic; 9 (18.8\%) were found deceased; 3 (6.2\%) had relocated; and 3 (6.2\%) were found to be missing. Among those patients not engaged in care, 4 were found to be acutely ill at the time of the home visit-demonstrating a severe exacerbation of their underlying mental illness and representing a potential harm to themselves. Measures were taken to transfer them to a hospital for additional evaluation and care. It was undetermined whether those who relocated were still engaged in care. Of those who had transferred care to a different clinic, the majority was reporting continuation of ART. The findings are summarized in Table 2.

Reasons for patients discontinuing follow-up, as collected during home visits, are shown in Table 3. Chief among these reasons was lack of transportation, distance from the clinic, and confusion regarding when to follow up.

Upon completion of the home visits patients were offered assistance reconnecting with the clinic if they were so inclined. Approximately 56\% of the patients who had previously been lost to follow up and successfully traced through home visits returned to the clinic after participation in the study and have maintained engagement in care as of July 31, 2013.

\section{Discussion}

In this study, we characterized the patients lost to follow up in an urban psychiatric HIV clinic in a resourcelimited setting. Over a 14-month period, nearly 1 in 4 patients receiving care had discontinued follow-up. This proportion is consistent with "best case scenario" estimates in previous large systematic reviews on retention in care in sub-Saharan Africa [22] [27] [28]. However, the studies included in those reviews were conducted over longer periods of time and separated individuals whether they were on ART or had not yet qualified. Although the number is still concerning, it is encouraging that persons with HIV and severe mental illness can be retained in care at similar rates to populations without mental illness, provided their care takes place in an integrated setting.

In comparison, for persons with mental illness and without HIV, a study conducted at the Chris Hani Baragwanath Academic Hospital followed 217 patients with schizophrenia over a 10-year period. Of those patients, 64\% did not adhere to their treatment and $62 \%$ of the study population had at least one relapse. Of those who were non-adherent to their medication, $80 \%$ had experienced a relapse [29] [30]. Interestingly, here, no integrated 
Table 1. Demographics.

\begin{tabular}{|c|c|}
\hline Study Variable & LTFU Total $(n=88$ \\
\hline Gender, \% female & 64.8 \\
\hline Age $^{\mathrm{a}}, \mathrm{y}$, mean (SD) & $37.2(10.1)$ \\
\hline Study Variable & LTFU Traced $(\mathbf{n}=4$ \\
\hline Gender, \% female & 62.5 \\
\hline Age $^{\mathrm{a}}, \mathrm{y}$, mean (SD) & $33.0(9.7)$ \\
\hline \multicolumn{2}{|l|}{ Ethnicity } \\
\hline Black & 100.0 \\
\hline \multicolumn{2}{|l|}{ Marital Status, n/N (\%) } \\
\hline \multicolumn{2}{|l|}{ Female } \\
\hline Single or divorced & $22 / 30(73.3)$ \\
\hline Married or partner & $8 / 30(26.7)$ \\
\hline \multicolumn{2}{|l|}{ Male } \\
\hline Single or divorced & $17 / 18(94.5)$ \\
\hline Married or partner & $1 / 18(5.5)$ \\
\hline \multicolumn{2}{|l|}{ Employment status, \% } \\
\hline Employed & 12.5 \\
\hline Unemployed & 87.5 \\
\hline \multicolumn{2}{|l|}{ TB history, n/N (\%) } \\
\hline History of TB & $18 / 48(37.5)$ \\
\hline No history of TB & $30 / 48(62.5)$ \\
\hline 44 count, cells $/ \mu \mathrm{L}$, median (IQR) & 143 (59 to 365$)$ \\
\hline
\end{tabular}

${ }^{\mathrm{a}}$ Age as of September 15, 2011.

Table 2. Reasons for LTFU.

\begin{tabular}{cc}
\hline Not engaged in care & n (\%) \\
Transfer of care & $21(43.8)$ \\
Receiving ART (8) & $12(25.0)$ \\
Not receiving ART (2) & \\
Not qualify for ART (2) & \\
Death & $9(18.8)$ \\
Relocated & $3(6.2)$ \\
Missing & $3(6.2)$ \\
\hline
\end{tabular}

care model was used and the study was conducted under routine outpatient circumstances. Other studies have shown that the medication compliance rate for non-psychiatric illness is $76 \%$, while that for psychiatric illness is $58 \%$ [31].

Tracing of patients discontinuing follow-up revealed that almost half of the individuals are not engaged in care, whereas one-quarter of patients have transferred their care to another clinic. This reaffirms that existing estimates of retention in care must be interpreted in light of specific contexts, definitions, and methods of ascertaining outcomes. Several of the reports on retention in care have been conducted from the perspective of the clinics-assuming that patients who are lost to follow up are no longer engaged in care. Our research demonstrated that a significant proportion of patients are still engaged in care and receiving ART, reflecting high mobility among HIV patients and ineffective health information systems that don't allow patients to be tracked 
Table 3. Reasons for not returning to care.

\begin{tabular}{cc}
\hline Causes for not returning* & n (\%) \\
\hline Money for transportation & $12(30.0)$ \\
Work/school schedule conflicting with follow-up & $6(15.0)$ \\
Distance for transportation & $5(12.5)$ \\
Confusion regarding follow-up (2-ART; 3-pre-ART) & $5(12.5)$ \\
Afraid to return secondary to shame/reprimanding & $3(7.5)$ \\
Surveillance fatigue & $2(5.0)$ \\
Hospitalization prevented follow-up & $2(5.0)$ \\
Felt well, so stopped ART & $1(2.5)$ \\
Toxicity, side effects, or adverse reactions & $1(2.5)$ \\
Money for food & $1(2.5)$ \\
Memory problems & $1(2.5)$ \\
Conflict with clinic/staff & $1(2.5)$ \\
\hline
\end{tabular}

*Some patients reported multiple reasons.

from site to site over time.

Interviews among those persons who did default from care showed a number of interesting findings. Chief among these was the fact that lack of transportation was the main reason persons reported for not being able to stay engaged in clinical care. Other structural barriers to maintaining engaged in care included distance from the clinic, confusion regarding their follow-up date, shame due to lack of follow-up, and conflict with work or school schedule. Interestingly, reasons cited for defaulting treatment in other studies of mentally ill patients have focused more on intolerance of side effects, relationship issues, and poor insight into their illness [30] [31].

Many programs aimed at improving retention in care often take an education-based approach [32]. This study suggests, however, that education about the importance of follow-up care may not be sufficient in and of itself to reduce loss of follow-up. Rather, programs must also address socioeconomic and structural barriers to staying in care in order to be successful. Addressing the financial burden of being engaged in care appears as an attractive target for intervention. Potential interventions include providing stipends or vouchers to patients to cover transportation costs, decentralization of services, or home-based care. Although not directly measured in this study, some patients who are unemployed do have access to pensions or disability grants. Therefore, further research is needed to address the total direct/indirect costs of being retained in care, and any availability of pensions, grants or supplementary income from partner/spouse or family.

\section{Limitations}

The study has multiple limitations. The first part of the study was a retrospective record review and subject to all the biases inherent in such methods. The second phase of the study collected data only on individuals who could be contacted and agreed to be in the study, and therefore cannot be generalized to the entire population who is lost to follow up. Also, reasons for being lost to follow up may differ among persons who could not be contacted or who refused to participate in the study. The study could have benefitted from a comparison between those lost to follow up and those retained in care, as well as a more complete characterization of those identified as lost to follow up, including further details on their history of mental illness. Due in part to human subjects restrictions in this vulnerable population and to problems with consistency in the larger clinic database, such comparisons were not possible. Future studies are being planned in this population to address these issues. Additionally, this study took place at an integrated clinic for persons with HIV and mental illness, and is thus not generalizable to populations with HIV and mental illness who do not receive integrated care. Finally, although not directly measured in our study, perceptions of stigma and associated discrimination relating to HIV, TB, and/or mental illness were highly suspected in our study population. There is strong evidence, including within South Africa that HIV/AIDS-related stigma serves as a major barrier to accessing prevention, care, and treatment services, including adherence to ART. 


\section{Conclusion}

In spite of these limitations, this study has several important findings. First, tracing patients through home visits proved to be an effective means to confirm the magnitude of patients lost to follow up, ascertain their outcomes, and elucidate the reasons for discontinuing care, as well as reengage them in care. Second, persons with severe mental illness and HIV can indeed be successfully retained in care for their HIV and mental health. There should be an increase in funding and advocacy for this dually diagnosed population to be treated, especially in settings that offer integrated care. Finally, the reasons that persons gave for not being able to continue in medical care were largely related to transportation barriers. This demonstrates the need for comprehensive services-including addressing socioeconomic barriers to care-to be provided in order to improve retention in medical care and ensure that persons have the best possible chance to live healthy lives with HIV.

\section{Conflict of Interests}

The authors of this study report no competing interests.

\section{Author's Contribution}

TW, GJ, and JF planned the study and wrote the manuscript. BG and YJ contributed to the manuscript and the overall study design. TW carried out the interviews and did the data analysis

\section{Acknowledgements}

We would like to acknowledge the Bristol Myers Squibb Foundation and Secure the Future and the Anova Health Institute for their ongoing technical support. We would also like to thank Peggy Motaung and Nomvula Bike for their assistance with the translation and home visits.

\section{References}

[1] Williams, B.G., Lima, V. and Gouws, E. (2011) Modeling the Impact of Antiretroviral Therapy on the Epidemic of HIV. Current HIV Research, 9, 367-382. http://dx.doi.org/10.2174/157016211798038533

[2] Mills, E.J., Nachega, J.B., Bangsberg, D.R., Singh, S., Rachlis, B., Wu, P., Wilson, K., Buchan, I., Gill, C.J. and Cooper, C. (2006) Adherence to HAART: A Systematic Review of Developed and Developing Nation Patient-Reported Barriers and Facilitators. PLoS Medicine/Public Library of Science, 3, e438.

[3] Gupta, A., Nadkarni, G., Yang, W.T., Chandrasekhar, A., Gupte, N., Bisson, G.P., Hosseinipour, M. and Gummadi, N. (2011) Early Mortality in Adults Initiating Antiretroviral Therapy (ART) in Low- and Middle-Income Countries (LMIC): A Systematic Review and Meta-Analysis. PLoS ONE, 6, e28691. http://dx.doi.org/10.1371/journal.pone.0028691

[4] Abdool Karim, S.S., Churchyard, G.J., Karim, Q.A. and Lawn, S.D. (2009) HIV Infection and Tuberculosis in South Africa: An Urgent Need to Escalate the Public Health Response. Lancet, 374, 921033.

[5] Munderi, P., Grosskurth, H., Droti, B. and Ross, D.A. (2012) What Are the Essential Components of HIV Treatment and Care Services in Low and Middle-Income Countries: An Overview by Settings and Levels of the Health System? AIDS, 26, S97-S103. http://dx.doi.org/10.1097/QAD.0b013e32835bdde6

[6] Sanne, I.M., Westreich, D., Macphail, A.P., Rubel, D., Majuba, P. and Van Rie, A. (2009) Long Term Outcomes of Antiretroviral Therapy in a Large HIV/AIDS Care Clinic In Urban South Africa: A Prospective Cohort Study. Journal of the International AIDS Society, 12, 1-11.

[7] Emamzadeh-Fard, S., Fard, S.E., Seyed Alinaghi, S. and Paydary, K. (2012) Adherence to Anti-Retroviral Therapy and Its Determinants in HIV/AIDS Patients: A Review. Infectious Disorders—Drug Targets, 12, 346-356. http://dx.doi.org/10.2174/187152612804142251

[8] http://www.health-e.org.za/uploaded/c0b24898135d6363351d590125ba7a6b.pdf

[9] Lane, T., Raymond, H.F., Dladla, S., Rasethe, J., Struthers, H., McFarland, W. and McIntyre, J. (2011) High Prevalence among Men Who Have Sex with Men in Soweto, South Africa: Results from the Soweto Men's Study. AIDS \& Behavior, 15, 626-634. http://dx.doi.org/10.1007/s10461-009-9598-y

[10] Senn, T.E. and Carey, M.P. (2009) HIV Testing among Individuals with a Severe Mental Illness: Review, Suggestions for Research, and Clinical Implications. Psychological Medicine, 39, 355-363. 
http://dx.doi.org/10.1017/S0033291708003930

[11] Bravo, P., Edwards, A., Rollnick, S. and Elwyn, G. (2010) Tough Decisions Faced by People Living with HIV: A Literature Review of Psychosocial Problems. AIDS Reviews, 12, 76-88.

[12] Whetten, K., Reif, S., Ostermann, J., Pence, B.W., Swartz, M., Whetten, R., Conover, C., Bouis, S., Thielman, N. and Eron, J. (2006) Improving Health Outcomes among Individuals with HIV, Mental Illness, and Substance Use Disorders in the Southeast. AIDS Care, 18, S18-26. http://dx.doi.org/10.1080/09540120600839330

[13] Collins, P.Y., Berkman, A., Mestry, K. and Pillai, A. (2009) HIV Prevalence among Men and Women Admitted to a South African Public Psychiatric Hospital. AIDS Care, 21, 863-867. http://dx.doi.org/10.1080/09540120802626188

[14] Weiser, S.D., Wolfe, W.R. and Bangsberg, D.R. (2004) The HIV Epidemic among Individuals with Mental Illness in the United States. Current HIV/AIDS Reports, 1, 186-192. http://dx.doi.org/10.1007/s11904-004-0029-4

[15] Sorsdahl, K.R., Mall, S., Stein, D.J. and Joska, J.A. (2011) The Prevalence and Predictors of Stigma amongst People Living with HIV/AIDS in the Western Province. AIDS Care, 23, 680-685. http://dx.doi.org/10.1080/09540121.2010.525621

[16] Walkup, J.T., Sambamoorthi, U. and Crystal, S. (2004) Use of Newer Antiretroviral Treatments among HIV-Infected Medicaid Beneficiaries with Serious Mental Illness. Journal of Clinical Psychiatry, 65, 1180-1189. http://dx.doi.org/10.4088/JCP.v65n0905

[17] Conover, C.J., Weaver, M., Ang, A., Arno, P., Flynn, P.M. and Ettner, S.L. (2009) HIV/AIDS Treatment Adherence, Health Outcomes and Cost Study. Costs of Care for People Living with Combined HIV/AIDS, Chronic Mental Illness, and Substance Abuse Disorders. AIDS Care, 21, 1547-1559. http://dx.doi.org/10.1080/09540120902923006

[18] Joska, J.A. and Sorsdahl, K.R. (2012) Integrating Mental Health into General Health Care: Lessons from HIV. African Journal of Psychiatry, 15, 420-423. http://dx.doi.org/10.4314/ajpsy.v15i6.52

[19] Moosa, M.Y.H., Jeenah, F.Y. and Kazadi, N. (2007) Treatment Adherence. South African Journal of Psychiatry, 13, 40-45.

[20] Himeloch, S., Brown, C.H. and Walkup, J. (2009) HIV Patients with Psychiatric Disorders Are Less Likely to Discontinue HAART. AIDS, 23, 1735-1742. http://dx.doi.org/10.1097/QAD.0b013e32832b428f

[21] Sorsdahl, K.R., Mall, S., Stein, D.J. and Joska, J.A. (2010) Perspectives towards Mental Illness in People Living with HIV/AIDS in South Africa. AIDS Care, 22, 1418-1427. http://dx.doi.org/10.1080/09540121003758655

[22] Fox, M.P. and Rosen, S. (2010) Patient Retention in Antiretroviral Therapy Programs up to Three Years on Treatment in Sub-Saharan Africa, 2007-2009: Systematic Review. Tropical Medicine \& International Health, 15, 1-15. http://dx.doi.org/10.1111/j.1365-3156.2010.02508.x

[23] Mugavero, M.J., Davila, J.A., Nevin, C.R. and Giordano, T.P. (2010) From Access to Engagement: Measuring Retention in Outpatient HIV Clinical Care. AIDS Patient Care \& Stds, 24, 607-613. http://dx.doi.org/10.1089/apc.2010.0086

[24] Delva, W., Eaton, J.W., Meng, F., Fraser, C., White, R.G., Vickerman, P., Boily, M.C. and Hallett, T.B. (2012) HIV Treatment as Prevention: Optimising the Impact of Expanded HIV Treatment Programmes. PLoS Medicine/Public Library of Science, 9, e1001258.

[25] Sahay, S., Reddy, K.S. and Dhayarkar, S. (2011) Optimizing Adherence to Antiretroviral Therapy. Indian Journal of Medical Research, 134, 835-849. http://dx.doi.org/10.4103/0971-5916.92629

[26] Jonsson, G., Furin, J., Jeenah, F., Moosa, M.Y., Sivepersad, R., Kalafatis, F. and Schoeman, J. (2011) Human Rights, Mental Illness, and HIV: The Luthando Neropsychiatric HIV Clinic in Soweto, South Africa. Health \& Human Rights, 13, E64-72.

[27] Rosen, S., Fox, M.P. and Gill, C.J. (2007) Patient Retention in Antiretroviral Therapy Programs in Sub-Saharan Africa: A Systematic Review. PLoS Medicine/Public Library of Science, 4, 1691-1701.

[28] Rosen, S. and Fox, M.P. (2011) Retention in HIV Care between Testing and Treatment in Sub-Saharan Africa: A Systematic Review. PLoS Medicine/Public Library of Science, 8, 1-16.

[29] Kazadi, N.J.B., Moosa, M.Y.H. and Jeenah, F.Y. (2008) Factors Associated with Relapse in Schizophrenia. South African Journal of Psychiatry, 14, 52-62.

[30] Awad, G.A. (2004) Antipsychotic Medications: Compliance and Attitude towards Treatment. Current Opinion in Psychiatry, 17, 75-80. http://dx.doi.org/10.1097/00001504-200403000-00002

[31] Ammassari, A., Trotta, M.P., Shalev, N., Marconi, P. and Antinori, A. (2012) Beyond Virological Suppression: The Role of Adherence in the Late HAART Era. Antiviral Therapy, 17, 785-792. http://dx.doi.org/10.3851/IMP2084

[32] Scanlon, M. and Vreeman, C. (2013) Current Strategies for Improving Access and Adherence to Antiretroviral Therapy in Resource-Limited Settings. HIV AIDS (Auckl), 5, 1-17. 